Homology, Homotopy and Applications, vol.18(2), 2016, pp.337-344

\title{
ON NONTRIVIALITY OF CERTAIN HOMOTOPY GROUPS OF SPHERES
}

\author{
SERGEI O. IVANOV, ROMAN MIKHAILOV AND JIE WU
}

(communicated by Graham Ellis)

\begin{abstract}
We provide an alternative proof of Gray's result that, for an odd prime $p$, there is a non-trivial $\mathbb{Z} / p$-component in the homotopy group $\pi_{(2 p-2) n+1}\left(S^{3}\right)$. As a corollary, it follows that, for $n \geqslant 2$, the homotopy groups $\pi_{n}\left(S^{2}\right)$ are non-zero.
\end{abstract}

\section{Introduction}

In [5], Curtis proved that $\pi_{n}\left(S^{4}\right) \neq 0$, for all $n \geqslant 4$. The main method from [5] of proving that a given element of the homotopy groups of spheres is non-zero is the analysis of Adams' $d$ and $e$-invariants of the stabilization of either that element or its Hopf image. This method allowed Curtis to prove that (see [5])

$$
\pi_{n}\left(S^{2}\right) \neq 0, n \neq 1 \bmod 8 .
$$

The same results on non-vanishing terms of the homotopy groups of spheres were obtained with the help of the composition method by Mimura, Mori and Oda [12].

Using the methods of the stable homotopy theory, the analysis of the image of the J-homomorphism and K-theory, it was shown by Mahowald [10, 11] and Mori [13] that

$$
\pi_{n}\left(S^{5}\right) \neq 0, n \geqslant 5
$$

On the other hand, since the fourth stable homotopy group of spheres is zero, one cannot get such a result for higher spheres, indeed $\pi_{n+4}\left(S^{n}\right)=0, n \geqslant 6$. The only remaining case to consider when such phenomena can happen is the case of $S^{2}$ and $S^{3}$. The main result of this paper is the following:

Theorem 1.1. For $n \geqslant 2$, the homotopy groups $\pi_{n}\left(S^{2}\right)$ are non-zero.

Since $\pi_{n}\left(S^{3}\right)=\pi_{n}\left(S^{2}\right), n \geqslant 3$, the same result follows for the homotopy groups $\geqslant 3$ of the 3 -sphere.

The main result (Theorem 1.1) is supported by the Russian Science Foundation, grant N 14-2100035. The last author is also partially supported by the Singapore Ministry of Education research grant (AcRF Tier 1 WBS No. R-146-000-222-112) and a grant (No. 11329101) of NSFC of China. Received January 10, 2016; published on November 18, 2016. 2010 Mathematics Subject Classification: Primary 55Q40; Secondary 55T15.

Key words and phrases: homotopy group, Lambda algebra, Toda element.

Article available at http://dx.doi.org/10.4310/HHA.2016.v18.n2.a18

Copyright (C) 2016, International Press. Permission to copy for private use granted. 
In the proof of Theorem 1.1, we cover the gaps in dimensions $\equiv 1 \bmod 8$ by showing that, for any odd prime $p$ and $n \geqslant 2$,

$$
\mathbb{Z} / p \subseteq \pi_{(2 p-2) n+1}\left(S^{3}\right) .
$$

In particular,

$$
\mathbb{Z} / 3 \subseteq \pi_{4 n+1}\left(S^{3}\right), \quad \mathbb{Z} / 15 \subseteq \pi_{8 n+1}\left(S^{3}\right) .
$$

Let $\pi_{k}^{n}$ denote the 2-component of $\pi_{k}\left(S^{n}\right)$. According to [5, Table on p. 543], the 2-component $\pi_{k}^{4} \neq 0$ for $k>4$. Mahowald [11, Theorem 1.6] and Mori [13, Corollary $5.12(\mathrm{iv})$ ] also proved the stronger statement that $\pi_{k}^{5} \neq 0$ for $k>5$. For the 2 -component $\pi_{k}^{3}$ of $\pi_{*}\left(S^{3}\right)$, Curtis proved that $\pi_{n}^{3} \neq 0, n \neq \equiv 1,2 \bmod 8$. The nontriviality of these cases can be also read from the fact that the 2 -local $v_{1}$-periodic homotopy group $v_{1}^{-1} \pi_{n}^{3} \neq 0$ if and only if $n \not \equiv 1,2 \bmod 8$ by [6, Theorem 4.2]. For the remaining cases of $\pi_{n}\left(S^{3}\right)$ with $n \equiv 1,2 \bmod 8$, notice that the 2-components of $\pi_{9}\left(S^{3}\right)$ and $\pi_{10}\left(S^{3}\right)$ both vanish, and so it is necessary to fulfil odd primes for having the non-triviality. Indicated from [15, Figure 3.3.18], one could have the following conjecture:

Conjecture. ${ }^{1}$ The 2-component of $\pi_{n}\left(S^{3}\right)$ is non-trivial for $n>10$.

After writing this paper, the authors became aware of the result from the paper [7]. The gaps in dimensions $\equiv 1 \bmod 8$ have been covered by a result of Gray [7, Theorem 12(e)] although the result of Theorem 1.1 is not mentioned in [7]. We point out that the method of the present paper for proving Theorem 1.1 is different from that in [7].

\section{Lambda-algebra and Toda elements}

Recall that, for any $k \geqslant 1$ and an odd prime $p$, the homotopy groups $\pi_{2(p-1) k+2}\left(S^{3}\right)$ contain non-trivial elements $\alpha_{k}(3)$ called the Toda elements. The elements $\alpha_{k}(3)$ have non-zero stable images in $\pi_{2(p-1) k-1}^{S}$. We will use the standard notation

$$
\alpha_{k}(m)=\Sigma^{m-3}\left(\alpha_{k}(3)\right) \in \pi_{2(p-1) k+m-1}\left(S^{m}\right), m \geqslant 3 .
$$

There exists a $p$-local EHP sequence

$$
J_{p-1}\left(S^{4}\right) \longrightarrow \Omega S^{5} \stackrel{H_{p}}{\longrightarrow} \Omega S^{4 p+1},
$$

where $J_{p-1}\left(S^{4}\right)$ is the $(2 p-1)$-skeleton of $\Omega S^{5}$, which implies the long exact sequence of homotopy groups $[\mathbf{1 7},(2.11)$, p. 103]

$$
\cdots \longrightarrow \pi_{n+1}\left(S^{4 p+1}\right) \stackrel{P}{\longrightarrow} \pi_{n-1}\left(J_{p-1}\left(S^{4}\right)\right) \stackrel{E}{\longrightarrow} \pi_{n}\left(S^{5}\right) \stackrel{H_{p}}{\longrightarrow} \pi_{n}\left(S^{4 p+1}\right) \longrightarrow \cdots .
$$

The following statement seems to be known. For example, there is a discussion of this result at the end of page 535 in [2]. However, we were not able to find an explicit reference to this statement and give here a proof.

\footnotetext{
${ }^{1}$ During the private circulation of this article, Doug Ravenel wrote a comment that it could be the case that all other 2-components of $\pi_{*}\left(S^{3}\right)$ are nontrivial except $\pi_{9}\left(S^{3}\right)$ and $\pi_{10}\left(S^{3}\right)$.
} 
Proposition 2.1. For $k \geqslant 2$, if the image of the map

$$
H_{p}: \pi_{2 k(p-1)+4}\left(S^{5}\right) \rightarrow \pi_{2 k(p-1)+4}\left(S^{4 p+1}\right)
$$

contains the element $\alpha_{k-2}(4 p+1)$, then $k \equiv 0 \bmod p$.

Let $p$ be a fixed odd prime number. The mod- $p$ lambda algebra ${ }_{[p]} \Lambda=\Lambda$ is an $\mathbb{F}_{p^{-}}$ algebra generated by elements $\lambda_{i}$ of degree $2(p-1) i-1$ for $i \geqslant 1$ and elements $\mu_{j}$ of degree $2(p-1) j$ for $j \geqslant 0$. We will use the following notations for $\mathrm{a}(k, j), \mathrm{b}(k, j) \in \mathbb{F}_{p}$

$$
\begin{aligned}
& \mathrm{a}(k, j)=(-1)^{j+1}\left(\begin{array}{c}
(p-1)(k-j)-1 \\
j
\end{array}\right), \\
& \mathrm{b}(k, j)=(-1)^{j}\left(\begin{array}{c}
(p-1)(k-j) \\
j
\end{array}\right),
\end{aligned}
$$

and for $N(k), N^{\prime}(k) \in \mathbb{Z}$ :

$$
N(k)=\left\lfloor k-\frac{k+1}{p}\right\rfloor, N^{\prime}(k)=\left\lfloor k-\frac{k}{p}\right\rfloor .
$$

The ideal of relations in $\Lambda$ is generated by the following relations:

$$
\begin{aligned}
& \lambda_{i} \lambda_{p i+k}=\sum_{j=0}^{N(k)} \mathrm{a}(k, j) \lambda_{i+k-j} \lambda_{p i+j}, i \geqslant 1, k \geqslant 0, \\
& \lambda_{i} \mu_{p i+k}=\sum_{j=0}^{N(k)} \mathrm{a}(k, j) \lambda_{i+k-j} \mu_{p i+j}+\sum_{j=0}^{N^{\prime}(k)} \mathrm{b}(k, j) \mu_{i+k-j} \lambda_{p i+j}, i \geqslant 1, k \geqslant 0, \\
& \mu_{i} \lambda_{p i+k+1}=\sum_{j=0}^{N(k)} \mathrm{a}(k, j) \mu_{i+k-j} \lambda_{p i+j+1}, i \geqslant 0, k \geqslant 0, \\
& \mu_{i} \mu_{p i+k+1}=\sum_{j=0}^{N(k)} \mathrm{a}(k, j) \mu_{i+k-j} \mu_{p i+j+1}, i \geqslant 0, k \geqslant 0 .
\end{aligned}
$$

The differential $\partial: \Lambda \rightarrow \Lambda$ is given by

$$
\begin{aligned}
\partial \lambda_{k} & =\sum_{j=1}^{N(k)} \mathrm{a}(k, j) \lambda_{k-j} \lambda_{j}, \\
\partial \mu_{k} & =\sum_{j=0}^{N(k)} \mathrm{a}(k, j) \lambda_{k-j} \mu_{j}+\sum_{j=1}^{N^{\prime}(k)} \mathrm{b}(k, j) \mu_{k-j} \lambda_{j} .
\end{aligned}
$$

Further by $\nu_{i}$ we denote an element of $\left\{\lambda_{i}, \mu_{i}\right\}$. A monomial $\nu_{i_{1}} \cdots \nu_{i_{l}}$ is said to be admissible if $i_{k+1} \leqslant p i_{k}-1$ whenever $\nu_{i_{k}}=\lambda_{i_{k}}$ and if $i_{k+1} \leqslant p i_{k}$ whenever $\nu_{i_{k}}=\mu_{i_{k}}$. The set of admissible monomials is a basis of $\Lambda$. The unstable lambda algebra $\Lambda(n)$ is a dg-subalgebra of $\Lambda$ generated by admissible elements $\nu_{i_{1}} \cdots \nu_{i_{l}}$ such that $i_{1} \leqslant n$. We denote by $\Lambda(n)_{m}$ the subspace generated by monomials of degree $m$ in $\Lambda(n)$ and 
by $\Lambda(n)_{m, l}$ the vector space generated by monomials of length $l$ in $\Lambda(n)_{m}$. Then

$$
\Lambda(n)=\bigoplus_{m, l} \Lambda(n)_{m, l}, \quad \Lambda(n)_{m}=\bigoplus_{l} \Lambda(n)_{m, l}
$$

Consider the left ideal $\Lambda \lambda=\sum_{i} \Lambda \lambda_{i}$ of $\Lambda$. The set of all admissible monomials $\nu_{i_{1}} \cdots \nu_{i_{l}}$ such that $\nu_{i_{l}}=\lambda_{i_{l}}$ forms a basis of $\Lambda \lambda$. Further we put

$$
\Lambda \lambda(n)=\Lambda \lambda \cap \Lambda(n) .
$$

There exists a spectral sequence which converges to the $p$-primary components of the homotopy groups of spheres, whose $E^{1}$-page is the lambda-algebra and $d^{1}$-differential is the differential in the lambda algebra:

$$
E^{1}(n)=\Lambda \lambda(n) \Rightarrow{ }_{(p)} \pi_{*}\left(S^{2 n+1}\right)
$$

This is an integral version of the well-known lower central series spectral sequence of six authors [3]. This spectral sequence was considered in details in the thesis of Leibowitz $[\mathbf{9}]$.

In the language of the lambda-algebra, the elements $\alpha_{k}$ can be presented as (see, for example, $[\mathbf{1 6}, 2.9]) \mu_{1}^{k-1} \lambda_{1}$. The map $H_{p}: \Omega S^{5} \rightarrow \Omega S^{4 p+1}$ induces a map $h_{p}$ on the level of $E^{1}$-terms of the spectral sequence (see [16, p. 23], and also $[\mathbf{1 9}, \mathbf{8}]$ ) with the short exact sequence

$$
\begin{gathered}
0 \rightarrow \Lambda(1) \oplus \lambda_{2} \Lambda(5) \longrightarrow \Lambda(2) \stackrel{h_{p}}{\longrightarrow} \Lambda(2 p) \rightarrow 0 \\
h_{p}\left(\mu_{1} \alpha\right)=h_{p}\left(\lambda_{1} \alpha\right)=h_{p}\left(\lambda_{2} \alpha\right)=0
\end{gathered}
$$

for any $\alpha$ and

$$
h_{p}\left(\mu_{2} \alpha\right)=\alpha \in \Lambda(4 p+1)
$$

Lemma 2.2. The linear map

$$
d_{1}: \operatorname{span}\left(\mu_{1}^{k} \lambda_{2},\left\{\mu_{1}^{k-i} \mu_{2} \mu_{1}^{i-1} \lambda_{1}\right\}_{i=1}^{k}\right) \longrightarrow \operatorname{span}\left(\left\{\mu_{1}^{k-i} \lambda_{1} \mu_{1}^{i} \lambda_{1}\right\}_{i=0}^{k}\right)
$$

is an isomorphism if and only if $k+2 \not \equiv 0(\bmod p)$.

Proof. Using the definition of $d_{1}=: d$ we get

$$
d\left(\lambda_{1}\right)=0, \quad d\left(\mu_{1}\right)=-\lambda_{1} \mu_{0}, \quad d\left(\lambda_{2}\right)=-2 \lambda_{1}^{2}, \quad d\left(\mu_{2}\right)=-\lambda_{2} \mu_{0}-2 \lambda_{1} \mu_{1}+\mu_{1} \lambda_{1} .
$$

Using the relations $\mu_{0} \mu_{1}=0=\mu_{0} \lambda_{1}$ and $\mu_{0} \lambda_{2}=-\mu_{1} \lambda_{1}, \mu_{0} \mu_{2}=-\mu_{1} \mu_{1}$, it is easy to compute that

$$
\begin{aligned}
& d\left(\mu_{2} \lambda_{1}\right)=-2 \lambda_{1} \mu_{1} \lambda_{1}+\mu_{1} \lambda_{1}^{2}, \\
& d\left(\mu_{1} \lambda_{2}\right)=\lambda_{1} \mu_{1} \lambda_{1}-2 \mu_{1} \lambda_{1}^{2}, \\
& d\left(\mu_{1} \mu_{2} \lambda_{1}\right)=\lambda_{1} \mu_{1}^{2} \lambda_{1}-2 \mu_{1} \lambda_{1} \mu_{1} \lambda_{1}+\mu_{1}^{2} \lambda_{1}^{2} .
\end{aligned}
$$

Moreover, we obtain $d\left(\mu_{1}\right) \mu_{1}=0$ and $d\left(\mu_{1}\right) \lambda_{1}=0$. It follows that

$$
\begin{aligned}
d\left(\mu_{1}^{k} \lambda_{2}\right) & =\mu_{1}^{k-1} d\left(\mu_{1} \lambda_{2}\right)=\mu_{1}^{k-1} \lambda_{1} \mu_{1} \lambda_{1}-2 \mu_{1}^{k} \lambda_{1}^{2}, \\
d\left(\mu_{1}^{k-1} \mu_{2} \lambda_{1}\right) & =\mu_{1}^{k-2} d\left(\mu_{1} \mu_{2} \lambda_{1}\right)=\mu_{1}^{k-2} \lambda_{1} \mu_{1}^{2} \lambda_{1}-2 \mu_{1}^{k-1} \lambda_{1} \mu_{1} \lambda_{1}+\mu_{1}^{k} \lambda_{1}^{2}, \\
d\left(\mu_{1}^{k-i-1} \mu_{2} \mu_{1}^{i} \lambda_{1}\right) & =\mu_{1}^{k-i-2} d\left(\mu_{1}\right) \mu_{2} \mu_{1}^{i} \lambda_{1}+\mu_{1}^{k-i-1} d\left(\mu_{2}\right) \mu_{1}^{i} \lambda_{1} \\
& =\mu_{1}^{k-i-2} \lambda_{1} \mu_{1}^{i+2} \lambda_{1}-2 \mu_{1}^{k-i-1} \lambda_{1} \mu_{1}^{i+1} \lambda_{1}+\mu_{1}^{k-i} \lambda_{1} \mu_{1}^{i} \lambda_{1},
\end{aligned}
$$


for $1 \leqslant i \leqslant k-2$ and

$$
d\left(\mu_{2} \mu_{1}^{k-1} \lambda_{1}\right)=d\left(\mu_{2}\right) \mu_{1}^{k-1} \lambda_{1}=-2 \lambda_{1} \mu_{1}^{k} \lambda_{1}+\mu_{1} \lambda_{1} \mu_{1}^{k-1} \lambda_{1} .
$$

If we denote $v_{i}:=\mu_{1}^{k-i} \lambda_{1} \mu_{1}^{i} \lambda_{1}$ for $0 \leqslant i \leqslant k$, and $u_{i}=\mu_{1}^{k-i} \mu_{2} \mu_{1}^{i-1} \lambda_{1}$ for $1 \leqslant i \leqslant k$ and $u_{0}=\mu_{1}^{k} \lambda_{2}$, then

$$
d\left(u_{0}\right)=v_{1}-2 v_{0}, \quad d\left(u_{i}\right)=v_{i+1}-2 v_{i}+v_{i-1}, \quad d\left(u_{k}\right)=-2 v_{k}+v_{k-1} .
$$

The matrix corresponding to this linear map is the following matrix:

$$
\left(\begin{array}{rrrrrr}
-2 & 1 & 0 & 0 & \cdots & 0 \\
1 & -2 & 1 & 0 & \cdots & 0 \\
0 & 1 & -2 & 1 & \ddots & 0 \\
\vdots & \ddots & \ddots & \ddots & \ddots & \vdots \\
0 & \cdots & 0 & 0 & 1 & -2
\end{array}\right) .
$$

It is easy to check by induction that its determinant is equal to $(-1)^{k+1}(k+2)$. It follows that $d: \operatorname{span}\left(u_{0}, \ldots, u_{k}\right) \rightarrow \operatorname{span}\left(v_{0}, \ldots, v_{k}\right)$ is an isomorphism if and only if $k+2 \not \equiv 0(\bmod p)$.

Now we are ready to prove Proposition 2.1.

Proof of Proposition 2.1. Indeed, if the Toda element $\alpha_{k-2}=\mu_{1}^{k-3} \lambda_{1}$ lies in the $H_{p}$ image, then there must be some term on $E^{2}$-page like $C \mu_{2} \mu_{1}^{k-3} \lambda_{1}+\sum \cdots, C \not \equiv$ $0 \bmod p$, which maps onto $\alpha_{k-2}$ by $h_{p}$. However, by Lemma 2.2 , this is possible only in the case $k \equiv 0 \bmod p$, in all other cases the corresponding $E^{2}$-term of the spectral sequence for $S^{5}$ is zero.

\section{3. $\quad$ Proof of Theorem 1.1}

For the proof of Theorem 1.1, we will use the following classical results.

(1) $[\mathbf{1}]$ or $\left[\mathbf{1 7},(4.3)\right.$, p. 112]. The element $\alpha_{k} \in \pi_{2(p-1) k-1}^{S}$ is not divisible by $p$ for $k \not \equiv 0 \bmod p$.

(2) Let $p>2$. By the classical work of Cohen, Moore and Neisendorfer [4], there exists a map $\pi: \Omega^{2} S^{2 n+1} \rightarrow S^{2 n-1}$ such that the composite

$$
\Omega^{2} S^{2 n+1} \stackrel{\pi}{\longrightarrow} S^{2 n-1} \stackrel{\Sigma^{2}}{\longrightarrow} \Omega^{2} S^{2 n+1}
$$

is homotopic to the $p$-th power map $p: \Omega^{2} S^{2 n+1} \rightarrow \Omega^{2} S^{2 n+1}$, where the case $p=3$ is given in [14, Theorem 4.1]. Following the notation in [4], let $D(n)$ be the homotopy fibre of $\pi: \Omega^{2} S^{2 n+1} \rightarrow S^{2 n-1}$. According to [4, Section 6], $D(p) \simeq \Omega^{2} S^{3}\langle 3\rangle$ and so there is a fibre sequence

$$
\Omega S^{2 p-1} \stackrel{\tau}{\longrightarrow} \Omega^{2} S^{3}\langle 3\rangle \stackrel{\theta}{\longrightarrow} \Omega^{2} S^{2 p+1} \stackrel{\pi}{\longrightarrow} S^{2 p-1}
$$

that implies a long exact sequence

$$
\cdots \longrightarrow \pi_{n+1}\left(S^{2 p+1}\right) \stackrel{\pi_{*}}{\longrightarrow} \pi_{n-1}\left(S^{2 p-1}\right) \stackrel{\tau_{*}}{\longrightarrow} \pi_{n}\left(S^{3}\right) \stackrel{\theta_{*}}{\longrightarrow} \pi_{n}\left(S^{2 p+1}\right) \longrightarrow \cdots,
$$

with the property that, for every $i$, the composition

$$
\pi_{i+2}\left(S^{2 p+1}\right) \stackrel{\pi_{*}}{\longrightarrow} \pi_{i}\left(S^{2 p-1}\right) \stackrel{\Sigma^{2}}{\longrightarrow} \pi_{i+2}\left(S^{2 p+1}\right)
$$


is the multiplication by $p$.

(3) $\left[\mathbf{1 7},(2.12)\right.$, p. 104]. For $m \geqslant 2$, denote by $Q_{2}^{2 m-1}$, the homotopy fibre of the double suspension map $S^{2 m-1} \rightarrow \Omega^{2} S^{2 m+1}$. We will use the notation from [17]. The natural map $Q_{2}^{2 m-1} \rightarrow S^{2 m-1}$ induces the map on homotopy groups $p_{*}$. There is a natural map

$$
I: \pi_{i}\left(Q_{2}^{2 m-1}\right) \rightarrow \pi_{i+3}\left(S^{2 m p+1}\right),
$$

such that the composition

$$
\pi_{i+3}\left(S^{2 m+1}\right) \rightarrow \pi_{i}\left(Q_{2}^{2 m-1}\right) \stackrel{I}{\longrightarrow} \pi_{i+3}\left(S^{2 m p+1}\right)
$$

is the Hopf map $H_{p}$.

For a given $k \not \equiv 1 \bmod p$, consider the element

$$
\alpha_{k-1} \in \pi_{2(p-1)(k-1)+2 p-2}\left(S^{2 p-1}\right)=\pi_{2(p-1) k}\left(S^{2 p-1}\right) .
$$

Suppose that $\alpha_{k-1}(2 p-1) \in i m\left\{\pi_{*}: \pi_{2(p-1) k+2}\left(S^{2 p+1}\right) \rightarrow \pi_{2(p-1) k}\left(S^{2 p-1}\right)\right\}$. Then the element $\Sigma^{2} \alpha_{k-1}(2 p-1)=\alpha_{k-1}(2 p+1)$ is $p$-divisible by $(2)$, hence its stable image is $p$-divisible. But this is not possible by (1). We conclude that

$$
\tau_{*}\left(\alpha_{k-1}(2 p-1)\right) \neq 0
$$

by the long exact sequence in (2), and so

$$
\mathbb{Z} / p \subseteq \pi_{2(p-1) k+1}\left(S^{3}\right), k \not \equiv 1 \bmod p .
$$

Now we recall the following statement of Toda ([17, Theorem $5.2(\mathrm{ii})]$, case $m=1)$. For $k \geqslant 2$, there exist an element

$$
\gamma^{\prime} \in \pi_{2 p+2 k(p-1)-1}\left(Q_{2}^{3}\right)=\pi_{2(p-1)(k+1)+1}\left(Q_{2}^{3}\right),
$$

such that

$$
I\left(\gamma^{\prime}\right)=\alpha_{k-1}(4 p+1) \in \pi_{4 p+2(k-1)(p-1)}\left(S^{4 p+1}\right) .
$$

Here $I: \pi_{2 p+2 k(p-1)-1}\left(Q_{2}^{3}\right) \rightarrow \pi_{2 p+2 k(p-1)+2}\left(S^{4 p+1}\right)$. Suppose that $p_{*}\left(\gamma^{\prime}\right)=0$, then

$$
\gamma^{\prime} \in \operatorname{im}\left\{H^{(2)}: \pi_{2(p-1)(k+1)+4}\left(S^{5}\right) \rightarrow \pi_{2(p-1)(k+1)+1}\left(Q_{2}^{3}\right)\right\} .
$$

In this case, we get

$$
\alpha_{k-1}(4 p+1) \in \operatorname{im}\left\{H_{p}: \pi_{2(k+1)(p-1)+4}\left(S^{5}\right) \rightarrow \pi_{2(k+1)(p-1)+4}\left(S^{4 p+1}\right)\right\} .
$$

This is possible only for $k+1 \equiv 0 \bmod p$ by Proposition 2.1. For $k+1 \not \equiv 0 \bmod p$, we get $0 \neq p_{*}\left(\gamma^{\prime}\right) \in \pi_{2(p-1)(k+1)+1}\left(S^{3}\right)$. Therefore,

$$
\mathbb{Z} / p \subseteq \pi_{2(p-1) k+1}\left(S^{3}\right), k \not \equiv 0 \bmod p .
$$

The statements $(A)$ and $(B)$ together give the needed statement:

$$
\mathbb{Z} / p \subseteq \pi_{2(p-1) k+1}\left(S^{3}\right), k \geqslant 1 .
$$

Theorem 1.1 now follows, since all dimensions $\equiv 1 \bmod 8$ are covered, moreover there is a $\mathbb{Z} / 15$-summand in homotopy groups $\pi_{8 l+1}\left(S^{2}\right), l \geqslant 2$.

As a final remark we observe that homotopy groups of $S^{2}$ in certain dimension $\equiv$ 1 mod 8 can be covered in another way. For that, we recall the results from $[\mathbf{1 7}, \mathbf{1 3}]$. 
(4) $\left[\mathbf{1 8}\right.$, Lemma 15.3(i)]. Let $y \in \pi_{i}\left(S^{2 p-1}\right)$ be an element of order $p$. There exists an element $a \in \pi_{i+2}\left(S^{3}\right)$, such that

$$
H_{p}(a)=x \Sigma^{2} y \in \pi_{i+2}\left(S^{2 p+1}\right),
$$

for some $x \not \equiv 0 \bmod p$.

(5) For $f \geqslant 0$, there is a family of elements $\alpha_{i}^{(f)} \in \pi_{2 i(p-1) p^{f}+2 f+2}\left(S^{2 f+3}\right)$ of order $p^{f}$, which have non-zero stable image in $\pi_{2 i(p-1) p^{f}-1}^{S}$. The $e$-invariants of these elements are the following: $e_{C}\left(\alpha_{i}^{(f)}\right)=-p^{-f-1}$.

(6) $[\mathbf{1 3}$, Lemma 4.1]. Let $f, g \geqslant 0, i, j \geqslant 1$ and

$$
\begin{aligned}
& \alpha: S^{2 n+2 i(p-1) p^{f}-1} \rightarrow S^{2 n}, \\
& \beta: S^{2 n+2 i(p-1) p^{f}+2 j(p-1) p^{g}-2} \rightarrow S^{2 n+2 i(p-1) p^{f}-1} .
\end{aligned}
$$

Assume that $e_{C}(\alpha) e_{C}(\beta)=p^{-u}$ and

$$
\begin{aligned}
& \nu_{p}(j)+g+1<u \leqslant \nu_{p}(i)+f+1+i(p-1) p^{f}, \\
& u+\nu_{p}\left(i p^{f}+j p^{g}\right)-\nu_{p}(i)-f-i(p-1) p^{f} \leqslant n<u+\nu_{p}\left(i p^{f}+j p^{g}\right)-\nu_{p}(j)-g,
\end{aligned}
$$

then $\alpha \circ \beta$ non-zero.

Now we will show that, for any $k \geqslant 1$, there is a non-zero $p$-torsion element in $\pi_{2(p-1)\left(p^{p} k+1\right)+1}\left(S^{3}\right)$. For that, consider the case $g=0, f=p-2, i=p^{2} k-1, j=$ $p^{p-2}$. By (6), we see that, $\alpha_{i}^{(p-2)} \circ \alpha_{j}^{(0)}$ is a non-zero element in the homotopy group $\pi_{2(p-1) p^{p} k+2 p-1}\left(S^{2 p+1}\right)$ which is equal to the image of the double suspension of an element of order $p$ from $\pi_{2(p-1) p^{p} k+2 p-3}\left(S^{2 p-1}\right)$. Hence, by (4), there is an element in $\pi_{2(p-1) p^{p} k+2 p-1}\left(S^{3}\right)=\pi_{2(p-1)\left(p^{p} k+1\right)+1}\left(S^{3}\right)$ whose $H_{p}$-image gives a non-zero multiple of this element.

\section{Acknowledgments}

The authors would like to thank F. Petrov for discussions related to the subject of the paper. The authors also wish to thank Martin Bendersky, Fred Cohen, Don Davis, Brayton Gray, Haynes Miller and Doug Ravenel most warmly for their important comments during the private circulation of this article.

\section{References}

[1] J.F. Adams, On the groups $J(X)$. IV. Topology 5 (1966), 21-71.

[2] M. Bendersky, Unstable towers in the odd primary groups of spheres, Trans. Amer. Math. Soc. 287 (1985), 529-542.

[3] A.K. Bousfield, E.B. Curtis, D.M. Kan, D.G. Quillen, D.L. Rector, J.W. Schlesinger, The mod- $p$ lower central series and the Adams spectral sequence, Topology 5 (1966), 331-342.

[4] F.R. Cohen, J.C. Moore, J.A. Neisendorfer, The double suspension and exponents of the homotopy groups of spheres, Ann. of Math. 110 (1979), no. 3, 549-565.

[5] E. Curtis, Some nonzero homotopy groups of spheres, Bull. Amer. Math. Soc. (N.S.) 75 (1969), 541-544. 
[6] D.M. Davis, Computing $v_{1}$-periodic homotopy groups of spheres and some compact Lie groups, Handbook of Algebraic Topology, 993-1048, North-Holland, Amsterdam, 1995.

[7] B. Gray, Unstable families related to the image of J, Math. Proc. Cambridge Philos. Soc. 96 (1984), no. 1, 95-113.

[8] J. Harper, H. Miller, On the double suspension homomorphism at odd primes, Trans. Amer. Math. Soc. 273 (1982), 319-331.

[9] D. Leibowitz, The $E^{1}$ term of the lower central series spectral sequence for the homotopy of spaces, PhD thesis, Brandeis University (1972).

[10] M. Mahowald, Description homotopy of the elements in the image of the Jhomomorphism, Manifolds Tokyo, 255-264, Univ. of Tokyo Press, 1975.

[11] M. Mahowald, The image of $J$ in the EHP sequence. Ann. of Math. (2) 116 (1982), no. 1, 65-112.

[12] M. Mimura, M. Mori, N. Oda, On the homotopy groups of spheres, Proc. Japan Acad. 50 (1974), 277-280.

[13] M. Mori, Applications of secondary e-invariants to unstable homotopy theory groups of spheres, Mem. Fac. Sci., Kyushu Univ. Ser. A 29 (1975), 59-87.

[14] J.A. Neisendorfer, 3-primary exponents, Math. Proc. Cambridge Philos. Soc. 90 (1981), 63-83.

[15] D.G. Ravenel, Complex Cobordism and Stable Homotopy Groups of Spheres, 2nd edn., AMS Chelsea Publishing, American Mathematical Society, 2003.

[16] M.C. Tangora, Computing the Homology of the Lambda Algebra, Mem. Amer. Math. Soc. 337 (1985), v+163 pp.

[17] H. Toda, On iterated suspensions I, J. Math. Kyoto Univ. 5 (1965), 87-142.

[18] H. Toda, On iterated suspensions III, J. Math. Kyoto Univ. 8 (1968), 101-130.

[19] R. Thompson, The $\nu_{1}$-periodic homotopy groups of an unstable sphere at odd primes, Trans. Amer. Math. Soc. 319 (1990), 535-559.

Sergei O. Ivanov ivanov.s.o.1986@gmail.com

Chebyshev Laboratory, St. Petersburg State University, 14th Line, 29b, St. Petersburg, 199178 Russia

Roman Mikhailov rmikhailov@mail.ru

Chebyshev Laboratory, St. Petersburg State University, 14th Line, 29b, St. Petersburg, 199178 Russia, and St Petersburg Department of Steklov Mathematical Institute 191023, 27 Fontanka, St. Petersburg, Russia

Jie $\mathrm{Wu}$ matwuj@nus.edu.sg

Department of Mathematics, National University of Singapore, 10 Lower Kent Ridge Road, Singapore 119076 